\title{
Prevalence of anelloviruses (TTV, TTMDV, and TTMV) in healthy blood donors and in patients infected with HBV or HCV in Qatar

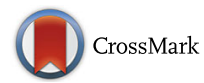

\author{
Ahmed A. Al-Qahtani ${ }^{1,2,3}$, Enas S. Alabsi ${ }^{4,5}$, Raed AbuOdeh ${ }^{6}$, Lukman Thalib ${ }^{4}$, Mohamed E El Zowalaty ${ }^{7}$
} and Gheyath K. Nasrallah ${ }^{4,5^{*}}$

\begin{abstract}
Background: Anelloviruses (TTV, TTMV, and TTMDV) have been associated with non A-G hepatitis. The goal of the current study was to estimate the prevalence of these anelloviruses in Qatar.

Methods: A total of 607 blood samples (500 healthy donors, and 53 HBV-and 54 HCV-positive patients) representing different nationalities were tested for the presence of TTV, TTMV, and TTMDV DNA by nested PCR.

Results: Prevalence rates for the three viruses were high in all studied groups, and exceeding 95\% in the HBV group (for TTV and TTMDV). Infection with more than one type of viruses was common and significant in most of the positive patients $(p<0.05)$ and ranging from 55.4\% for TTV/TTMV and TTMV/TTMDV co-infections in the healthy group, to $96.3 \%$ for TTV/TTMV co-infections in the HBV group. Further, and as with most previous studies, no significant association was found between anelloviruses infections and age, nationality, or gender $(p>0.05)$ albeit the detection of higher infection rates among females and Qatari subjects.

Conclusion: This was the first published study to look at prevalence of Anellowviruses in the Middle East. High prevalence rates of the three viruses in all studied groups was noted. Further studies are needed to explore and compare the different genotypes of these viruses in the region.
\end{abstract}

Keywords: Anelloviruses, HBV, HCV, PCR

\section{Background}

Human infections with anelloviruses (small ssDNA viruses) are widely spread [1, 2]. A prominent member of the Anelloviridae family is the Torque teno virus (TTV), reported for the first time in 1997 in a Japanese patient with post-transfusion hepatitis of unknown etiology [3]. Subsequently (in 2000), another small DNA virus, designated Torque teno mini virus (TTMV), remotely similar to TTV was discovered [4]. In 2007 a third addition to the anellovirus genus was reported; the new virus had a genome of $3.2 \mathrm{~kb}$ (TTV and TTMV were around 3.8 and $2.8 \mathrm{~kb}$, respectively), hence the designation Torque

\footnotetext{
* Correspondence: gheyath.nasrallah@qu.edu.qa

${ }^{4}$ Department Health Sciences, College of Arts and Sciences, Qatar University, PO Box 2713, Doha, Qatar

${ }^{5}$ Biomedical Research Center, Qatar University, Doha, Qatar

Full list of author information is available at the end of the article
}

teno midi virus (TTMDV) [5]. The Anelloviridae family contains 12 genera; TTV, TTMV, and TTMDV belong to Alphatorquevirus, Betatorquevirus, and Gammatorquevirus, genera respectively [6].

The Alphatorquevirus genera (TTV) alone has 29 species. Much less is known about phylogenetic groups for TTMV and TTMDV [7]; TTMV ORF1 sequence comparison showed significant divergence, and complete genome analysis of TTMDV also shows sequences clustering [7].

The three viruses share a similar genome organization (UTR region, open reading frame region followed by short G/C rich region). Further, the three viruses are extremely divergent in the ORF regions both at the nucleotide and amino acid levels [6].

Furthermore, infection with anelloviruses can be highly common reaching a $90 \%$ prevalence rate [8]. Reports on the prevalence of TTMV in humans indicated high rates of infection in French [9], Brazilian [10, 11] 
and in Norwegian [12] blood donors and also in French hemodialysis patients (95\%) [13]. Further, TTMV DNA has been detected in amniotic fluid, cord blood and breast milk $[10,14]$. TTMV DNA has also been detected in cervical swabs of healthy women [15], women diagnosed with cervical cancers, peripheral blood mononuclear cells, feces and saliva [16].

TTMDV DNA was detected in plasma and saliva samples of French blood donors [17], in healthy and hepatitis C Italian serum samples [18], in healthy blood donors, hepatitis $\mathrm{C}$ patients and HIV patients in Iran [19], and in Korean chronic hepatitis patients and blood products [20]. TTMDV was also reported in Hungarian children with acute respiratory disease [21].

Since TTV was first detected in blood samples it was referred to as transfusion-transmitted (TT) virus [3]. Subsequent studies suggested the existence of other ways of TTV transmission including parenteral, sexual, vertical and others [22].

TTV has been detected in almost every human tissue type or body fluid, the newly discovered TTMV can be transmitted from mother to baby; similar virus sequences in mother and baby were detected in umbilical cord blood, breast milk, amniotic fluid, and sera of newborns [7].

Finally, viremic infections with multiple genotypes of any of the three viruses at any one time is not uncommon $[12,17,23]$.

The aim of the current study was to investigate the rates of infection of TTV, TTMV, and TTMDV in Qatar, and to examine the association of these virus infections, if any, with $\mathrm{HBV}$ or $\mathrm{HCV}$.

\section{Methods}

\section{Ethical approval}

Prior to samples collection from patients and blood donors, Institutional Review Board of Hamad Medical Corporation (HMC) (Protocol No.13422/13) and Qatar University (IRB No. QU-IRB289-A/14) approvals were obtained. Furthermore, because this research involves no risk to the participants, a waiver of all the consent requirements was obtained. The participants' rights and welfare were not harmfully affected, because personal information related to participants was not claimed. Blood samples obtained from the blood bank and the hospital were anonymous. The only information collected related to the participants were nationality, sex, and age. Information concerning collected data was kept confidential.

\section{Blood samples collection}

The samples that were used in this project to detect TTMDV and TTMV DNA were exactly the same ones we used in our previous TTV published project [8], and the data from it were used again in this study for comparison purposes between the three anelloviruses.

A total of 500 samples from healthy blood donors were used in the present study. These samples were collected from the blood donation unit during the period from June to August 2013. In addition, blood samples from 54 infected $\mathrm{HBV}$ to $53 \mathrm{HCV}$ patients were also obtained from the diagnostic virology laboratory at the HMC.

\section{Plasma DNA extraction}

DNA was extracted from $200 \mu \mathrm{l}$ aliquots of plasma using a standard commercial kit for viral DNA extraction from body fluids (PureLink ${ }^{\circ}$ Viral RNA/DNA Kits - Life Technologies; Grand Island, New York, USA).

\section{Whole genome amplification of DNA}

Due to small sample volume, extracted DNA was amplified using REPLI-g Mini Kit (Catalog \# 150025, Qiagen, USA) following manufacturer's protocol. Amplified DNA was stored at $-20{ }^{\circ} \mathrm{C}$ until use.

\section{Detection of TTMDV and TTMV}

Both viruses were detected using nested PCR. For the first round PCR setup, the following components were used: one $\mu \mathrm{l}$ of whole genome amplified DNA, $10 \mathrm{pmol}$ each of forward and reverse primers (universal primers) as described previously [24] and GoTaq Green Master Mix (Promega, Madison, WI, USA) in a reaction volume of $50 \mu \mathrm{l}(\mu \mathrm{l})$. The mixture was preheated at $95^{\circ} \mathrm{C}$ for $5 \mathrm{~min}$ followed by 35 cycles with parameters as follows: $95^{\circ} \mathrm{C}$ for $30 \mathrm{~s}, 55^{\circ} \mathrm{C}$ for $30 \mathrm{~s}, 72^{\circ} \mathrm{C}$ for $30 \mathrm{~s}$, and a final extension of $72{ }^{\circ} \mathrm{C}$ for $5 \mathrm{~min}$. For the second round, two PCR reactions to detect TTMDV and TTMV were separately prepared. For the detection of TTMDV, the reaction mixture consisted of $5 \mu \mathrm{l}$ of the amplification product of the first round, 15 pmol of NG795/NG796 primers [24] and GoTaq Green Master Mix with a total volume of $40 \mu \mathrm{l}$. The mixture was preheated at $95^{\circ} \mathrm{C}$ for 5 min followed by 30 cycles of $95^{\circ} \mathrm{C}$ for $20 \mathrm{~s}, 62^{\circ} \mathrm{C}$ for $20 \mathrm{~s}, 72^{\circ} \mathrm{C}$ for $20 \mathrm{~s}$, and a final extension of $72^{\circ} \mathrm{C}$ for $5 \mathrm{~min}$. The same conditions were used to detect TTMV except that the primers NG792/NG793/NG794 as sense primers and NG791 as antisense primer were used [24]. Fifteen $\mu \mathrm{l}$ of the amplified products were electrophoresed in $2.5 \%$ agarose gel, stained with GreenView Plus (GeneCopoeia, USA), and photographed under UV light.

\section{Statistical analysis}

Data were analyzed using SPSS software. The chi-square test was used to compare proportions between the groups. Differences were considered to be statistically significant at was $p<0.05$. 


\section{Results}

\section{Study population analysis}

In the present study, a total of 607 plasma samples were screened for the presence of anellovirsuses (TTV, TTMDV, TTMV) DNA using nested PCR. The majority of samples were from healthy blood donors and nonQatari male nationals. Age, gender and nationality distributions were not significantly different in healthy blood donors and HBV- or HCV-positive patients. Blood donors in Qatar were predominantly males, and so did those tested positive for $\mathrm{HBV}$ and $\mathrm{HCV}$. It should be noted that the expatriate population makes up the vast majority of residents in Qatar with about only $16 \%$ of those residing in Qatar are Qatari nationals. Our study population reflects the demographic distribution in the population. In terms of age, it appears that the majority of the HBV patients were younger than 30 years whilst the majority of $\mathrm{HCV}$ patients were older than 50 years as was shown in Table 1.

\section{Anelloviruses infection rates in the different populations} In order to determine the rate of infection of the three anelloviruses, plasma DNA was extracted from a total of 607 blood samples representing healthy blood donors $(n=500)$, HBV $(n=54)$, and HCV $(n=53)$-infected patients residing in Qatar, and DNA of each virus was screened by nested PCR as described in the Methods. Figure 1 depicts the prevalence of TTV, TTMDV and TTMV DNA among the overall studied population. The overall prevalence of TTV DNA $(85.2 \%)$ was significantly $(p<0.001)$ higher than TTMDV (76.3\%), and TTMV (66.6\%). Further, TTV prevalence was significantly $(p<0.001)$ higher among the healthy donor population than TTMDV and TTMV (Table 2).

We also noticed that patients co-infected with HBV or HCV showed significantly $(p<0.05)$ higher infection rates with anelloviruses than the healthy blood donors. The only exception was in the insignificant association between TTV and HCV infection. A closer look at Table 2 revealed that the highest prevalence of the three

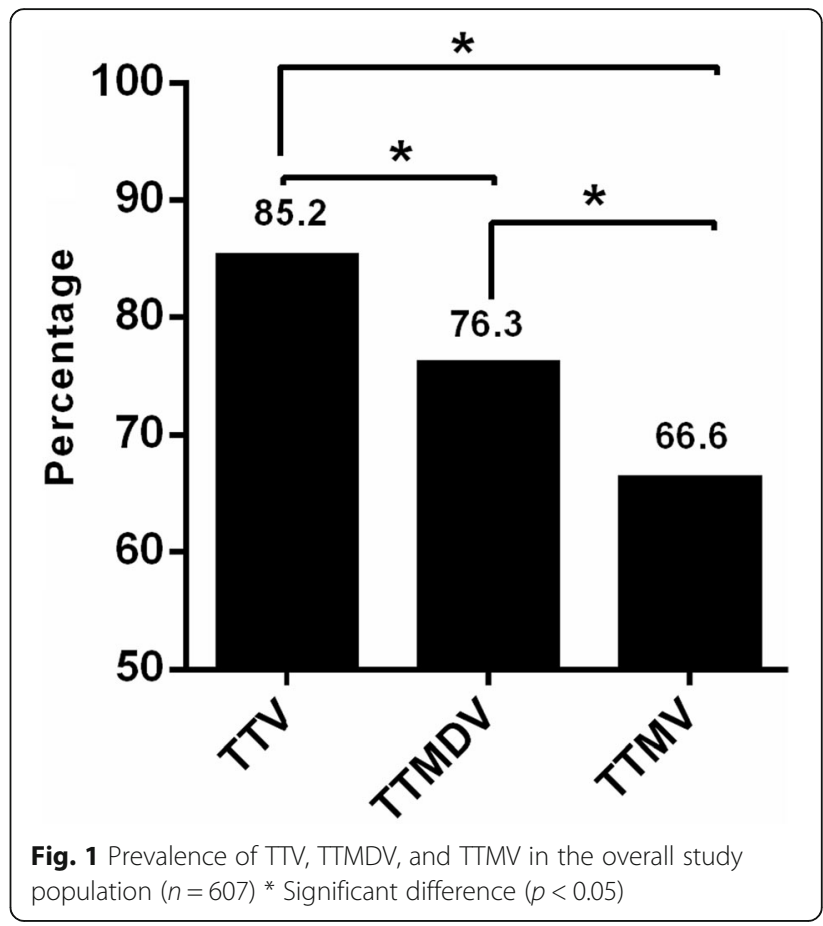

viruses was among the HBV followed by the HCV- infected patients.

\section{Analysis of mixed infections}

Our data also showed that co-infections between the three anelloviruses was also very common (Table 3). The study found that $67.2 \%$ of healthy blood donors were infected with both TTV and TTMDV $(p<0.001)$, and $55.4 \%$ were infected with TTV and TTMV or TTMDV and TTMV $(p<0.001)$. The co-infection rates were significantly higher in blood donors $(p<0.001)$; there was no significant association between infections with any of TTV and TTMDV, TTV and TTMV or TTMDV and TTMV among HBV or HCV-infected patients.

Table 1 Characteristics of the subjects $(n=607)$

\begin{tabular}{|c|c|c|c|c|}
\hline & & Healthy $(n=500) n(\%)$ & $\mathrm{HBV}(n=54) n(\%)$ & $\mathrm{HCV}(n=53) n(\%)$ \\
\hline \multirow[t]{2}{*}{ Gender } & Male & $488(97.6)$ & $39(72.2)$ & $39(73.6)$ \\
\hline & Female & $12(2.4)$ & $15(27.8)$ & $14(26.4)$ \\
\hline \multirow[t]{2}{*}{ Nationality } & Qatari & $60(12.0)$ & $9(16.7)$ & $12(22.6)$ \\
\hline & Non-Qatari & $440(88.0)$ & $45(83.3)$ & $41(77.4)$ \\
\hline \multirow[t]{4}{*}{ Age (years) } & $<30$ & $122(24.4)$ & $22(40.7)$ & $9(17.0)$ \\
\hline & $31-40$ & $227(45.4)$ & $18(33.3)$ & $9(17.0)$ \\
\hline & $41-50$ & $108(21.6)$ & $6(11.1)$ & $12(22.6)$ \\
\hline & $>51$ & $43(8.6)$ & $8(14.8)$ & $23(43.4)$ \\
\hline Age range (Average $\pm S D$ ) & $13-80(36 \pm 10.6)$ & $19-74(36 \pm 9.3)$ & $13-74(34.9 \pm 12.0)$ & $15-80(45 \pm 14.9)$ \\
\hline
\end{tabular}


Table $2 T V, T M V$, and TTMDV distribution in study groups

\begin{tabular}{llllll}
\hline & Healthy $(n=500)$ & HBV $(n=54)$ & $p$-value * & HCV $(n=53)$ & $n(\%)$ \\
\hline$n(\%)$ & $n(\%)$ & $52(96.3)$ & $<0.01$ & $46(86.8)$ & $47(88.7)$ \\
TTV & $417(83.4)$ & $53(98.1)$ & $<0.001$ & $44(83.0)$ & 0.52 \\
TTMDV & $373(74.6)$ & $46(85.2)$ & $<0.001$ & 0.69 \\
TTMV & $306(61.2)$ & 0.04 & & \\
\hline *** $p$-value & $<0.001$ & & & \\
\hline
\end{tabular}

* Healthy vs HBV, ** Healthy vs HCV, ${ }^{* * *}$ TTV vs TTMDV vs TTMV

The distribution of infection with TTV, TTMDV, or TTMDV among different nationalities, ages, and gender was shown in Table 4; there was no significant association between infection with TTV, TTMDV, or TTMDV and age or gender.

Furthermore, since the expatriate population makes up the vast majority of residents in Qatar, we sought to study the distribution of the three anelloviruses in different nationalities residing in Qatar (Tables 2 and 5). As shown in Table 2, although the Qatari population had the highest prevalence of the three anelloviruses, the difference was not statistically significant.

Further, as shown in Table 5, there was no significant differences in the prevalence of TTV, TTMDV, and TTMV among the different ethnic or nationality groups residing in Qatar, which further suggests that nationalities or population origin has no effect on the prevalence of these viruses in Qatar.

\section{Discussion}

Since their discovery, the three anelloviruses TTV, TTMV, and TTMDV shared some common features such as their detection in the blood of healthy donors and in $\mathrm{HBV} / \mathrm{HCV}$ pationts. The presence of the viruses in non- A to $G$ hepatitis patients, linked them to hepatitis. This probably justifies the number of studies, specially on TTV, that evaluated the prevalence of the virus in HBV and HCV blood samples compared to the limited number of studies in healthy individuals especially in the Middle East region. In a previous study [8] we reported our finding concerning the prevalence of TTV in healthy blood donors and HBV/HBC patients in Qatar. Therefore, the objective of this study was to evaluate the

Table 3 Association between TTV, TTMV, and TTMDV in different study populations

\begin{tabular}{llccccc}
\hline & & TTMDV $n(\%)$ & $p$-value & TTMV $n(\%)$ & $p$-value \\
\hline Healthy $(n=500)$ & TTV & $336(67.2)$ & $<0.001$ & $277(55.4)$ & $<0.001$ \\
& TTMDV & & - & $277(55.4)$ & $<0.001$ \\
HBV $(n=54)$ & TTV & $46(85.2)$ & 0.14 & $52(96.3)$ & 0.03 \\
& TTMDV & & - & $46(85.2)$ & 0.02 \\
HCV $(n=53)$ & TTV & $41(77.4)$ & 0.05 & $43(81.1)$ & 0.02 \\
& TTMDV - & - & $39(73.6)$ & 0.33 \\
\hline
\end{tabular}

TTMV and TTMDV prevalence rates in healthy, as well as, HBV and HCV subjects in Qatar and compare the findings to those in the previous TTV study.

The current study was conducted on a total of 607 blood samples from healthy blood donors $(n=500)$, subjects with HBV $(n=54)$ or HBC $(n=53)$. Findings of the present study showed an overall high prevalence rates of the three viruses (Fig. 1); TTV prevalence rates $(85.2 \% \mathrm{t}$ ) were significantly higher $(p<0.05)$ than the other two viruses $(76.3$ and $66.6 \%$ for TTMDV, and TTMV, respectively). A closer comparative look at the ditribution of the three viruses in the different study groups (healthy, $\mathrm{HBV}$, and $\mathrm{HCV}$ ) revealed interesting findings. Firstly, the incidences of the three viruses were significantly high in the HBV and HCV groups as compared to the healthy group $(p<0.05)$ (Table 2); prevalence rates were even highre in the HBV group (reaching 98\% in the HBV group for TTMDV). Secondly, the prevalence rates of the three viruses were significantly different in the healthy $(p<0.001)$ and HBV $(p<0.04)$ groups but not in the HCV group (Table 2).

Further, most of the tested samples, regarless of the study group, yielded mixed infections; the highest association was seen in the HBV group between TTV and TTMV (96.3\% of the samples); most of the associations were significant $(p<0.05)$ except for the mixed TTV/ TTMDV infecitons in the HBV group and TTMDV/ TTMV mixed infections in the HCV group (Table 3).

Prevalence rates for the three viruses were not significantly different in relation to gender, nationality or age (Table 4) $(p>0.05)$, albeit the slightly higher infection rates in females and Qatari nationals. A closer look at the overall distribution of the viruses in different ethnic backgrounds as compared to Qatari nationals revealed higher rates in Qataris and "others" compared to Asians and Africans $(p>0.05)$ (Table 5).

There is great prevalence variation of anelloviruses in different regions of the world. This variability can be explained in different ways; it is probably a true representation of the ubiquity of the viruses; it can be also attributed to the choice of DNA target region amplified and PCR primers used.

The high prevalence rates of anelloviruses viremias in this study, and other studies for that matter, can be also 
Table 4 TV, TTMV, and TTMDV distribution in study groups $(n=607)$

\begin{tabular}{|c|c|c|c|c|c|c|c|c|}
\hline & & Total $(n)$ & TTV n (\%) & $p$-value & TTMDV n (\%) & $p$-value & TTMV n (\%) & $p$-value \\
\hline \multirow[t]{2}{*}{ Gender } & Male & 566 & 47 (84.6) & 0.11 & $428(75.6)$ & 0.10 & 375 (66.3) & 0.34 \\
\hline & Female & 41 & $38(92.7)$ & & $35(85.4)$ & & $29(70.7)$ & \\
\hline \multirow[t]{2}{*}{ Nationality } & Qatari & 81 & $73(90.1)$ & 0.11 & $67(82.7)$ & 0.09 & $58(71.6)$ & 0.18 \\
\hline & Non-Qatari & 526 & $444(84.4)$ & & $396(75.3)$ & & $346(65.8)$ & \\
\hline \multirow[t]{4}{*}{ Age (Years) } & $<30$ & 153 & $134(87.6)$ & 0.45 & $107(69.9)$ & 0.06 & $97(63.4)$ & 0.25 \\
\hline & $31-40$ & 254 & $217(85.4)$ & & $204(80.3)$ & & $171(67.3)$ & \\
\hline & $41-50$ & 126 & $102(81.0)$ & & $92(73.0)$ & & $80(63.5)$ & \\
\hline & $>51$ & 74 & $64(86.5)$ & & $60(81.1)$ & & $56(75.7)$ & \\
\hline
\end{tabular}

explained in part, by the ease of transmission of the virus.

Compared to TTV, much less is known about the prevalence rates of the TTMV and TTMDV in healthy individuals, as well as HBV and HBC patients. Earlier reports on the prevalence of TTMV in other regions indicated comperable infection rates in blood donors from France (76-77\%) [9], Brazil (72-77\%) [10, 11] and Norway (48\%) [12]. Furthermore, lower prevalence rates were previously reported in healthy blood donors in Korea (41.3\%) [25] and Iran (17\%) [26].

With regards to TTMDV, the viremia frequency of the virus in healthy individuals was $14.5 \%$ in Iran [19], 34.5\% in Korea [27], 20\% in France [27], and 8.6\% in Italian blood donors [18]. Those findings are much lower than the $74.6 \%$ reported in the current study in Qatar.

Due to the scarcity of data regarding the incidence of TTMV and TTMDV in HBV- or HCV-positive subjects, it became more difficult to make any comparative analysis. An earlier study in Iran reported incidence rate of $20 \%(5 / 25)$ and $48 \%(12 / 25)$ of TTMV in HBV and HCV subjects [26]. Further, Garcia-Alvarez et al. reported a very high incidence rate of TTV and TTMV in HIV/ $\mathrm{HCV}$-coinfected patients, which is higher than the $83 \%$ infection rate in HCV patients in the current study [28]. Also, in another study in Iran, researchers reported a TTMDV incidence rate of $80.5 \%(29 / 36)$ and $75 \%(3 / 4)$ in $\mathrm{HIV} / \mathrm{HCV}$ and $\mathrm{HIV} / \mathrm{HCV} / \mathrm{HBV}$ coinfected patients, respectively [19], which was lower than the 88.7 and 98.1\% TTMDV infection rates obtained in HCV and HBV infected patients in the current study (Table 2).

Table 5 TTV, TTMV, and TTMDV distribution in different nationalities $(n=607)$

\begin{tabular}{llllll}
\hline & $\begin{array}{l}\text { Qatar } \\
(=81)\end{array}$ & $\begin{array}{l}\text { Asia } \\
(n=345)\end{array}$ & $\begin{array}{l}\text { Africa } \\
(n=156) \\
n(\%)\end{array}$ & $\begin{array}{l}\text { Others } \\
(n=25)\end{array}$ & $p$-value \\
& $n(\%)$ & $1 \%)$ & \\
\hline TTV & $73(90.1)$ & $294(85.2)$ & $127(81.4)$ & $23(92.0)$ & 0.23 \\
TTMDV & $67(82.7)$ & $265(76.8)$ & $109(69.9)$ & $22(88.0)$ & 0.06 \\
TTMV & $58(71.6)$ & $223(64.6)$ & $105(67.3)$ & $18(72.0)$ & 0.60 \\
\hline
\end{tabular}

High frequencies of TTMV and TTMDV among HBV and $\mathrm{HBC}$ patients in comparison to low frequencies in healthy blood donors suggest common infection routes, highly compatible with these viruses, such as blood and sex.

Moreover, coinfection with multiple TT viruses seems to be a common event [28-30]. One explanation for the mixed infections with different anelloviruses seen in the current study and other studies would be that the viruses may have similar and probably synergystic infection cycles that help viruses from the same family better adapt to the environment. Another explanation would be that these viruses probably infect the same cell types permitting the viruses to favorably replicate in the same cells.

\section{Conclusions}

In conclusion, this study showed a high prevalence $(>75 \%)$ of the three anelloviruses (TTV, TTMDV, and TTMV) in the Middle East. Further studies are needed to explore and compare the different genotypes of these viruses in the region.

\section{Acknowledgements}

We are grateful to: Mr. Tameem Hadwan, Mr. Abdullfattah Nasrallah, and Mrs. Mooza A. Al-Kinji and the students Aisha Khan, Nadima Ali, and Maria Khalid Samati for their technical support. We also would like to thank Ms. Sana M. Abohasera and Mrs. Faten H. Sawali from the Blood Donor Center at HMC for their help in blood sample and donor demographic data collection. This report (publication) was made possible by UREP grant \# (UREP 15-015-3-006) from the Qatar National Research Fund (a member of Qatar Foundation). The statements made herein are solely the responsibility of the author(s). This study was approved by the Hamad Medical Center Research Committee (Protocol \#13422/13).

\section{Funding}

This work was made possible by UREP grant \# (UREP 15-015-3-006) from the Qatar National Research Fund (a member of Qatar Foundation).

\section{Authors' contributions}

GNK and ESA collected samples, extracted DNA and prepared figures and tables. RA did the write up and revisions. LT performed the statistical analyses and data interpretation, MEZ helped in data entry, draw figure 1, and in table's constructions, and AAA conducted all PCR experiments of all samples. Further, GKN and RA supervised the entire work. All authors read and approved the final manuscript. 


\section{Competing interests}

The authors declare that they have no competing interests.

\section{Ethics approval and consent to participate}

Prior to samples collection from patients and blood donors, Institutional Review Board of Hamad Medical Corporation (HMC) (Protocol No.13422/13) and Qatar University (IRB No. QU-IRB289-A/14) approvals were obtained. The participants' rights and welfare were not harmfully affected, because personal information related to participants was not claimed. Blood samples obtained from the blood bank and the hospital were anonymous. The only information collected related to the participants were nationality, sex, and age. Information concerning collected data was kept confidential.

\section{Author details}

${ }^{1}$ Department of Infection and Immunity, Research Center, King Faisal Specialist Hospital and Research Center, Riyadh, Saudi Arabia. ${ }^{2}$ Department of Microbiology and Immunology, Alfaisal University School of Medicine, Riyadh, Saudi Arabia. ${ }^{3}$ Liver Disease Research Center, King Saud University, Riyadh, Saudi Arabia. ${ }^{4}$ Department Health Sciences, College of Arts and Sciences, Qatar University, PO Box 2713, Doha, Qatar. ${ }^{5}$ Biomedical Research Center, Qatar University, Doha, Qatar. ${ }^{6}$ Department of Medical Laboratory Sciences, University of Sharjah, Sharjah, UAE. 'Virology and Microbiology Research Laboratory \& Antibiotic Research Unit, School of Health Sciences, University of KwaZulu Natal, University Rd, Westville Campus, Durban 4000, South Africa.

\section{Received: 17 June 2016 Accepted: 2 December 2016}

Published online: 28 December 2016

\section{References}

1. Hino S, Miyata H. Torque teno virus (TTV): current status. Rev Med Virol. 2007; $17: 45-57$

2. Simmonds P, Davidson F, Lycett C, Prescott LE, MacDonald DM, Ellender J, Yap PL, Ludlam CA, Haydon GH, Gillon J, Jarvis LM. Detection of a novel DNA virus (TTV in blood donors and blood products. Lancet. 1998;352:191-5.

3. Nishizawa T, Okamoto H, Konishi K, Yoshizawa H, Miyakawa Y, Mayumi M. A novel DNA virus (TTV) associated with elevated transaminase levels in posttransfusion hepatitis of unknown etiology. Biochem Biophys Res Commun. 1997:241:92-7.

4. Takahashi K, Iwasa Y, Hijikata M, Mishiro S. Identification of a new human DNA virus (TTV-like mini virus, TLMV) intermediately related to $\Pi$ virus and chicken anemia virus. Arch Virol. 2000;145:979-93.

5. Ninomiya M, Nishizawa T, Takahashi M, Lorenzo FR, Shimosegawa T, Okamoto $\mathrm{H}$. Identification and genomic characterization of a novel human torque teno virus of $3.2 \mathrm{~kb}$. J Gen Virol. 2007;88:1939-44.

6. Biagini P. Classification of TTV and related viruses (anelloviruses). Curr Top Microbiol Immunol. 2009;331:21-33.

7. Spandole S, Cimponeriu D, Berca LM, Mihaescu G. Human anelloviruses: an update of molecular, epidemiological and clinical aspects. Arch Virol. 2015;160:893-908.

8. AbuOdeh R, Al-Mawlawi N, Al-Qahtani AA, Bohol MF, Al-Ahdal MN, Hasan HA, AbuOdeh L, Nasrallah GK. Detection and genotyping of torque teno virus (TTV) in healthy blood donors and patients infected with HBV or HCV in Qatar. J Med Virol. 2015;87:1184-91.

9. Biagini $P$, Gallian $P$, de Micco $P$, de Lamballerie $X$. TT virus and $T \pi$ virus-like mini-virus infection in french blood donors. Transfusion. 2000;40:1542.

10. Vasconcelos HC, Cataldo M, Niel C. Mixed infections of adults and children with multiple TTV-like mini virus isolates. J Med Virol. 2002;68:291-8.

11. Niel C, Lampe E. High detection rates of TTV-like mini virus sequences in sera from Brazilian blood donors. J Med Virol. 2001;65:199-205.

12. Moen EM, Huang L, Grinde B. Molecular epidemiology of TTV-like mini virus in Norway. Arch Virol. 2002;147:181-5.

13. Gallian P, Biagini $P$, Attoui $H$, Cantaloube JF, Dussol B, Berland $Y$, de Micco P, de Lamballerie $X$. High genetic diversity revealed by the study of TLMV infection in French hemodialysis patients. J Med Virol. 2002;67:630-5.

14. Matsubara H, Michitaka K, Horiike N, Kihana T, Yano M, Mori T, Onji M. Existence of TT virus DNA and TTV-like mini virus DNA in infant cord blood: mother-to-neonatal transmission. Hepatol Res. 2001;21:280-7.

15. Fornai C, Maggi F, Vatteroni ML, Pistello M, Bendinelli M. High prevalence of $\Pi$ virus (TTV) and TTV-like minivirus in cervical swabs. J Clin Microbiol. 2001;39:2022-4.
16. Changani $L$, Bouzari $M$, Talebi A. Torque teno mini virus infection in chronic cervicitis and cervical tumors in Isfahan, Iran. Intervirology. 2013;56:265-70.

17. Biagini $P$, de Micco $P$, de Lamballerie $X$. Identification of a third member of the Anellovirus genus ("small anellovirus") in French blood donors. Arch Virol. 2006;151:405-8.

18. Andreoli E, Maggi F, Pistello M, Meschi S, Vatteroni M, Nelli LC, Bendinelli M. Small Anellovirus in hepatitis $C$ patients and healthy controls. Emerg Infect Dis. 2006;12:1175-6.

19. Fatholahi M, Bouzari M. Torque Teno Midi Virus/Small Anellovirus in Sera of healthy, HIV/HCV and HIV infected individuals in Lorestan Province, Iran. Jundishapur J Microbiol. 2015:8:e25368.

20. Han Th CJY. Detection of small anellovirus DNA from blood products. Kore Blood Transfus. 2006;17:126-34

21. Burian Z, Szabo H, Szekely G, Gyurkovits K, Pankovics P, Farkas T, Reuter G. Detection and follow-up of torque teno midi virus ("small anelloviruses") in nasopharyngeal aspirates and three other human body fluids in children. Arch Virol. 2011;156:1537-41.

22. Vasilyev EV, Trofimov DY, Tonevitsky AG, llinsky W, Korostin DO, Rebrikov DV. Torque Teno Virus (TTV) distribution in healthy Russian population. Virol J. 2009:6:134.

23. Ninomiya M, Takahashi M, Nishizawa T, Shimosegawa T, Okamoto $\mathrm{H}$. Development of PCR assays with nested primers specific for differential detection of three human anelloviruses and early acquisition of dual or triple infection during infancy. J Clin Microbiol. 2008;46:507-14.

24. Ninomiya M, Takahashi M, Shimosegawa T, Okamoto $H$. Analysis of the entire genomes of fifteen torque teno midi virus variants classifiable into a third group of genus Anellovirus. Arch Virol. 2007;152:1961-75.

25. Chung JY, Han TH, Seong K, Paik IK, Kim MJ. Transfusion-transmitted virus and TTV-like mini virus infection in blood donors. Korean J Lab Med. 2004 28:250-54.

26. Ghazimorad A, Bouzari M, Kardi MT. Frequency of Torque Teno Mini Virus in Hepatitis B and C Patients and Healthy Blood Donors in Isfahan, Iran. Iranian Journal of Blood and Cancer. 2014:6:119-26.

27. Chung JY, Han TH, Koo JW, Kim SW, Seo JK, Hwang ES. Small anellovirus infections in Korean children. Emerg Infect Dis. 2007:13:791-3.

28. Garcia-Alvarez M, Berenguer J, Alvarez E, Guzman-Fulgencio M, Cosin J, Miralles P, Catalan P, Lopez JC, Rodriguez JM, Micheloud D, et al. Association of torque teno virus (TTV) and torque teno mini virus (TTMV) with liver disease among patients coinfected with human immunodeficiency virus and hepatitis C virus. Eur J Clin Microbiol Infect Dis. 2013:32:289-97.

29. Niel C, de Oliveira JM, Ross RS, Gomes SA, Roggendorf M, Viazov S. High prevalence of $\Pi$ virus infection in Brazilian blood donors. J Med Virol. 1999:57:259-63.

30. Luo K, He H, Liu Z, Liu D, Xiao H, Jiang X, Liang W, Zhang L. Novel variants related to $\Pi$ virus distributed widely in China. J Med Virol. 2002;67:118-26.

\section{Submit your next manuscript to BioMed Central and we will help you at every step:}

- We accept pre-submission inquiries

- Our selector tool helps you to find the most relevant journal

- We provide round the clock customer support

- Convenient online submission

- Thorough peer review

- Inclusion in PubMed and all major indexing services

- Maximum visibility for your research

Submit your manuscript at www.biomedcentral.com/submit
) Biomed Central 